\title{
Search for time lag in intra-day variability of $\operatorname{Sgr} \mathrm{A}^{*}$
}

\author{
A. Miyazaki ${ }^{1}$, S. S. Lee ${ }^{1}$, B. W. Sohn ${ }^{1}$, T. Jung ${ }^{1}$, \\ M. Tsuboi ${ }^{2}$ and T. Tsutsumi ${ }^{3}$ \\ ${ }^{1}$ Korea Astronomy and Space Science Institute, \\ 776 Daedeokdae-ro, Yuseong-gu, Daejeon 305-348, Republic of Korea \\ email: amiya@kasi.re.kr \\ ${ }^{2}$ Institute of Space and Astronautical Science, Japan Aerospace Exploration Agency, \\ 3-1-1 Yoshinodai, Chuo-ku, Sagamihara, Kanagawa 252-5210, Japan \\ ${ }^{3}$ National Radio Astronomy Observatory, \\ 1003 Lopezville Road, Socorro, NM 87801-0387, USA
}

\begin{abstract}
We searched the time lag between the intra-day variables (IDVs) of Sagittarius A* at 22,43 , and $86 \mathrm{GHz}$ bands using the Korean VLBI Network (KVN). The time lags between the IDV flare peaks at 22 and $43 \mathrm{GHz}$ are reported, and they suggest that the flare emissions come from adiabatically expanding plasma blobs, ejected close to the Galactic center black hole. We searched the time lags between light curves at 90 and $102 \mathrm{GHz}$ using the Nobeyama Millimeter Array, but could not find significant time lags. In order to detect the diversity of the time lags of $\mathrm{Sgr} \mathrm{A}^{*}$ flares, we performed observations of $\mathrm{Sgr} \mathrm{A}^{*}$ in the 22,43 , and $86 \mathrm{GHz}$ bands using the KVN in the winter of 2013. Because the receiver system of KVN can observe Sgr A* in these three bands simultaneously, the KVN is very useful to detect the time lags of Sgr A* flares.
\end{abstract}

Keywords. Galaxy: center — galaxies: nuclei — black hole physics

\section{Introduction}

Sagittarius A* $\left(\operatorname{Sgr} \mathrm{A}^{*}\right)$ is a compact source with emission from radio to X-ray, and it is believed to be associated with the Galactic center black hole (GCBH). After its discovery, Sgr A* was observed at many wavelengths, and temporal flux variations were reported. Time variability observation is a powerful tool to probe the structure and emission mechanisms. Radio flux monitoring of Sgr A* has been useful to explore the region around the GCBH. From $\mathrm{mm}$ to sub-mm wave, Intra-Day Variability (IDV), flux variability with a timescale of a few hours, has been observed frequently (e.g., Miyazaki et al. 2004, Mauerhan et al. 2005, Li et al. 2009). The emitter of Sgr A* is in controversy but undoubtedly the emission comes from very near the GCBH. The VLA detected time lags of 20-30 min between IDVs at 22 and $43 \mathrm{GHz}$ (Yusef-Zadeh et al. 2006, 2008). These time lags strongly suggest that the radio flux is emitted from adiabatically expanding plasma. However, truly simultaneous observations at both frequencies were performed in only one epoch. For verifying such observations, additional simultaneous observations are needed.

\section{Nobeyama Millimeter Array}

We performed the observations of the flux densities of Sgr A* at 90 and $102 \mathrm{GHz}$ in order to detect the time lag between these frequencies using the Nobeyama Millimeter Array (NMA) in Japan. We detected a radio flare during the observation on 2005 April 6, 


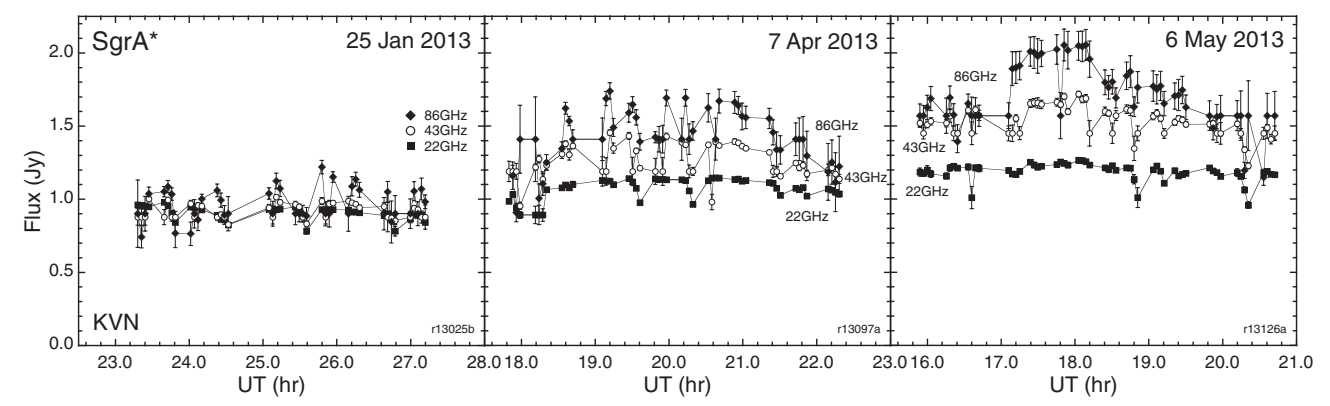

Figure 1. Light curves of Sgr A* observed by the KVN at 22, 43, and $86 \mathrm{GHz}$ simultaneously on Jan 25, Apr 7, and May 6, 2013. Filled squares, open circles, and filled diamonds indicate flux densities at 22,43 , and $86 \mathrm{GHz}$, respectively. The integration time of each data is $3 \mathrm{~min}$.

and calculated the $z$-transformed discrete correlation function (ZDCF; Alexander 1997) between the light curves. The time lag between these frequencies was not distinctly detected from the calculated ZDCF (Miyazaki et al. 2013). If the expanding plasma model, which explains the time lag at lower frequencies, is valid, the light curve at $90 \mathrm{GHz}$ would be delayed with respect to the one at $102 \mathrm{GHz}$. However, the estimated time lag with NMA is sufficiently smaller than the expected value. This result suggests that the plasma blobs ejected near the GCBH may be widely diverse especially in optical thickness (Miyazaki et al. 2013).

\section{Korean VLBI Network}

The Korean VLBI Network (KVN) is a three-element VLBI network with 21-m radio telescopes located in Seoul (Yonsei telescope), Ulsan (Ulsan telescope) and Jeju island (Tanma telescope) of South Korea. To enable multi-frequency observations, the KVN quasi-optics system splits the signal from the sub-reflector into four, using three dichroic low-pass filters. These signals in four different frequency bands are guided to corresponding receivers. Thus the receiver system of the KVN can observe in these four bands of $22,43,86$, and $129 \mathrm{GHz}$ simultaneously. The system is very useful in order to detect the time lag of Sgr A* flares. To search for the time lags of Sgr A* flares, we performed the observations of Sgr A* in the 22,43 , and $86 \mathrm{GHz}$ bands using the KVN in the winter of 2013. We constructed the six observations and made the light curves of Sgr A*. Figure 1 shows the light curves of Sgr A* at these bands using the KVN. Unfortunately, the Sgr A* flare in these three bands was not distinctly seen in these observations, and the time lag between the light curves was not clear. We will continue the Sgr A* multi-frequencies observations using KVN in the autumn of 2013.

\section{References}

Alexander, T. 1997, in D. Maoz, A. Sternberg, \& E. Leibowitz (eds.), Astronomical Time Series (Dordrecht: Kluwer), 163

Li, J., Shen, Z.-Q., Miyazaki, A., et al. 2009, ApJ, 700, 417

Mauerhan, J. C., Morris, M., Walter, F., \& Baganoff, F. K. 2005, ApJ, 623, L25

Miyazaki, A., Tsutsumi, T., \& Tsuboi, M. 2004, ApJ, 611, L97

Miyazaki, A., Tsuboi, M., \& Tsutsumi, T. 2013, PASJ, 65, L6

Yusef-Zadeh, F., Roberts, D., Wardle, M., Heinke, C. O., \& Bower, G. C. 2006, ApJ, 650, 189

Yusef-Zadeh, F., Wardle, M., Heinke, C., et al. 2008, ApJ, 682, 361 